\title{
Adubação de aveia em dois sistemas de plantio(1)
}

\author{
Ana Cândida Primavesi(2), Odo Primavesi(3), Heitor Cantarella ${ }^{(4)}$, Rodolfo Godoy(3) \\ e Lúcio José Vivaldi(5)
}

\begin{abstract}
Resumo - O objetivo deste trabalho foi determinar as doses de N, P e K necessárias para a obtenção da máxima produção econômica de forragem de aveia, linhagem UPF 87111, nos sistemas de plantio convencional e com cobertura morta, num Latossolo Vermelho distrófico típico. Utilizou-se como esquema experimental um fatorial fracionado (1/2) $4^{3}$ com dois blocos ao acaso, total de 32 parcelas, sem repetição. Os tratamentos constituíram-se de quatro doses de $\mathrm{N}$ e de $\mathrm{K}_{2} \mathrm{O}\left(0,70,140\right.$ e $\left.210 \mathrm{~kg} \mathrm{ha}^{-1}\right)$, como uréia e cloreto de potássio, respectivamente, e quatro doses de $\mathrm{P}_{2} \mathrm{O}_{5}\left(0,60,120,180 \mathrm{~kg} \mathrm{ha}^{-1}\right)$, como superfosfato triplo. As doses de $\mathrm{N}, \mathrm{Pe} \mathrm{K}$ para produção de forragem de aveia com máxima receita líquida, foram, em kg ha-1 $165(\mathrm{~N}), 50\left(\mathrm{P}_{2} \mathrm{O}_{5}\right), 53\left(\mathrm{~K}_{2} \mathrm{O}\right)$, no plantio convencional, e $210(\mathrm{~N}), 90\left(\mathrm{P}_{2} \mathrm{O}_{5}\right)$ no plantio com cobertura morta, com produções de matéria seca de, respectivamente, $6.641 \mathrm{~kg} \mathrm{ha}^{-1} \mathrm{e}$ $7.322 \mathrm{~kg} \mathrm{ha}^{-1}$. Nos dois sistemas de plantio houve resposta somente em relação ao N, e o seu uso resultou maior produção de forragem por unidade de nutriente aplicado.
\end{abstract}

Termos para indexação: Avena sativa, cultivo convencional, matéria vegetal, forragem, rendimento.

\section{Oat fertilization in two planting systems}

\begin{abstract}
The objective of this work was to determine the best rates of N, P, and K to obtain maximum economic return per area of forage yield for the oat line UPF 87111 grown in two planting systems i.e conventional tillage and with a corn-residue mulch. The experiments were carried out in a Dark-Red Latosol (Hapludox). The experimental design was a incomplete (1/2) $4^{3}$ factorial, with two randomized blocks, with a total of 32 plots, without replication. The treatments comprised four rates of $\mathrm{N}_{\text {and }} \mathrm{K}_{2} \mathrm{O}$ $\left(0,70,140\right.$, and $\left.210 \mathrm{~kg} \mathrm{ha}^{-1}\right)$, using urea and potassium chloride, respectively, and four rates of $\mathrm{P}_{2} \mathrm{O}_{5}(0$, 60,120 and $180 \mathrm{~kg} \mathrm{ha}^{-1}$ ), using triple superphosphate. The $\mathrm{N}, \mathrm{P}, \mathrm{K}$ rates for maximum profit were, in $\mathrm{kg} \mathrm{ha}^{-1}$ for conventional tillage: $\mathrm{N}=165, \mathrm{P}_{2} \mathrm{O}_{5}=50, \mathrm{~K}_{2} \mathrm{O}=53$; and for mulch-covered field: $\mathrm{N}=210$, $\mathrm{P}_{2} \mathrm{O}_{5}=90$, resulting in forage yields of, respectively, 6,641 and 7,322 kg ha-1 of dry matter. In both planting systems there was response only to $\mathrm{N}$, and its use resulted in higher forage yield per unit of applied fertilizer.
\end{abstract}

Index terms: Avena sativa, conventional tillage, mulches, forage, yields.

\footnotetext{
(1) Aceito para publicação em 19 de agosto de 2002.

(2) Embrapa-Centro de Pesquisa de Pecuária do Sudeste (CPPSE), Caixa Postal 339, CEP 13560-970 São Carlos, SP. E-mail: anacan@cppse.embrapa.br

(3) Embrapa-CPPSE. Bolsista do CNPq. E-mail: odo@cppse.embrapa.br, godoy@cppse.embrapa.br

(4) Instituto Agronômico, Centro de Solos e Recursos Agroambientais, Caixa Postal 28, CEP 13001-970 Campinas, SP. Bolsista do CNPq. E-mail: hcantare@barao.iac.br

${ }^{(5)}$ Universidade de Brasília, Instituto de Ciências Exatas, Caixa Postal 4481, CEP 70910-900 Brasília, DF. E-mail: vivaldi@unb.br
}

\section{Introdução}

O cultivo de aveia forrageira como alternativa de alimentação animal no período do inverno seco na Região Sudeste é bastante promissor, por sua boa produtividade e alto valor nutritivo.

Na Embrapa-Centro de Pesquisa de Pecuária do Sudeste estão sendo desenvolvidos estudos de manejo de cortes (Primavesi et al., 1999, 2000a, 2000b) e de adubação, com cultivares de aveia recomendadas por este centro, com a finalidade de formar um 
pacote tecnológico. A expansão da prática do plantio direto na palha e a necessidade de comparação com métodos convencionais geram a demanda de informação regional a este respeito. Um dos aspectos importantes do plantio direto é o recobrimento do solo com resíduos de palha que provoca modificação do microclima em relação às plantas cultivadas (Sediyama \& Prates, 1986), por causa da grande influência no regime hidrotérmico do solo, conservando a umidade diretamente, reduzindo a evaporação e aumentando a infiltração de água no solo. Além disso, controla indiretamente as ervas daninhas, o que pode reduzir a frequiência de irrigações no cultivo de aveia. Como a cobertura morta também reduz a temperatura, além de apresentar outros efeitos na biologia do solo e na disponibilidade de nutrientes, sua presença torna os primeiros centímetros do solo um ambiente mais adequado biologicamente, com maior desenvolvimento de raízes superficiais (Resende, 1986), podendo causar modificações na resposta das plantas à adição de nutrientes via adubação.

A linhagem de aveia UPF 87111 vem se destacando na avaliação e seleção de linhagens de aveia forrageira para serem lançadas como novas cultivares (Godoy et al., 1999).

O objetivo deste trabalho foi determinar as doses de N, P e K necessárias para obtenção da máxima produção econômica de forragem de aveia, linhagem UPF 87111, nos sistemas de plantio convencional e com cobertura morta, num Latossolo Vermelho distrófico típico.

\section{Material e Métodos}

Os experimentos foram realizados num Latossolo Vermelho distrófico típico (LVd), na fazenda Canchim, da Embrapa-Centro de Pesquisa de Pecuária do Sudeste, situada em São Carlos, SP, latitude $22^{\circ} 01^{\prime} \mathrm{S}$, e longitude 47054' W e altitude de $836 \mathrm{~m}$. O clima é tropical de altitude, com déficit hídrico no período de outono-inverno, ou seja, período no qual os experimentos foram realizados.

$\mathrm{O}$ experimento com plantio convencional recebeu $25 \mathrm{~mm}$ de água parcelados em duas irrigações por semana, estabelecidas com base na exigência de $4 \mathrm{~mm}$ de água por dia pela aveia forrageira, de acordo com Bacchi et al. (1996). $\mathrm{O}$ experimento usando plantio com cobertura morta recebeu $12,5 \mathrm{~mm}$ de água por semana, irrigação preestabelecida supondo-se uma perda menor de água do solo em virtude da cobertura do solo (Sediyama \& Prates, 1986).

Utilizou-se como esquema experimental um fatorial fracionado (1/2) $4^{3}$ com dois blocos ao acaso, num total de 32 parcelas, sem repetição, de acordo com Andrade \& Noleto (1986). Os tratamentos constaram de quatro doses de $\mathrm{N}\left(0,70,140\right.$ e $\left.210 \mathrm{~kg} \mathrm{ha}^{-1}\right)$, na forma de uréia; quatro doses de $\mathrm{P}_{2} \mathrm{O}_{5}\left(0,60,120,180 \mathrm{~kg} \mathrm{ha}^{-1}\right)$, como superfosfato triplo; e quatro doses de $\mathrm{K}_{2} \mathrm{O}\left(0,70,140\right.$ e $\left.210 \mathrm{~kg} \mathrm{ha}^{-1}\right)$, como cloreto de potássio. As doses de $\mathrm{P}_{2} \mathrm{O}_{5}$ foram aplicadas totalmente na semeadura (16/5/2000) e as doses de $\mathrm{N}$ e de $\mathrm{K}_{2} \mathrm{O}\left(70,140\right.$ e $\left.210 \mathrm{~kg} \mathrm{ha}^{-1}\right)$ foram parceladas respectivamente na semeadura, em 16/5/2000 $(10,20$ e $\left.30 \mathrm{~kg} \mathrm{ha}^{-1}\right)$, no perfilhamento, em 9/6/2000 (15, $30 \mathrm{e}$ $\left.45 \mathrm{~kg} \mathrm{ha}^{-1}\right)$, após o primeiro corte, em 7/7/2000 (15, 30 e $45 \mathrm{~kg} \mathrm{ha}^{-1}$ ), e após os cortes de rebrota, em 11/8/2000 e $15 / 9 / 2000\left(15,30\right.$ e $\left.45 \mathrm{~kg} \mathrm{ha}^{-1}\right)$.

As áreas dos experimentos eram contíguas e amostras de solo foram coletadas na profundidade de $0-20 \mathrm{~cm}$, em 11/11/1999. Não foi necessária a aplicação de calcário. A área destinada ao plantio convencional foi mantida em pousio por um ano, sendo roçada e rastelada em 12/4/2000; em 13/4/2000 foi feita nova coleta de solo da área. Dia 19/4/2000 realizou-se gradagem. Em 12/5/2000 foi passada enxada rotativa e quatro dias depois foram realizados adubação e plantio. $\mathrm{Na}$ área destinada ao plantio de aveia com cobertura morta, a cultura anterior foi o milho, sem adubação. Em 6/4/2000 foram colhidas as espigas de milho e dia 10/4/2000 coletaram-se novas amostras de solo. Os restos culturais foram cortados e pesados $\left(5.620 \mathrm{~kg} \mathrm{ha}^{-1}\right.$ de matéria seca) e em seguida picados e redistribuídos uniformemente na área que não recebeu qualquer revolvimento mecânico além da abertura dos sulcos para o plantio da aveia. As amostras de solos foram analisadas conforme Raij et al. (1987) (Tabela 1).

Em 16/5/2000 foi feito o plantio da aveia. As parcelas tinham cinco linhas de $6 \mathrm{~m}$ espaçadas de $20 \mathrm{~cm}$ e área útil de $3 \mathrm{~m}^{2}$. A semeadura foi feita manualmente, com $70 \mathrm{se}-$ mentes viáveis por metro linear, em sulcos com $3 \mathrm{~cm}$ de profundidade. O material de aveia usado, linhagem UPF 87111, é caracterizado como de ciclo longo e alta produtividade de forragem. Os cortes das plantas foram manuais, a 7-10 cm da superfície do solo. O manejo de cortes usado foi determinado por Primavesi et al. (2001), e possibilita a maior produção de matéria seca de aveia com as melhores características nutritivas. $O$ primeiro corte foi efetuado quando $10 \%$ das plantas iniciaram a elongação do colmo, e os três cortes de rebrota com intervalos de 35 dias.

Após a pesagem da matéria fresca da parcela, uma amostra com $500 \mathrm{~g}$ foi secada a $60^{\circ} \mathrm{C}$ em estufa com circulação forçada de ar por 48 horas, até peso constante, para determi- 
Tabela 1. Resultados da análise química do Latossolo Vermelho distrófico típico em três profundidades, anteriores à instalação dos experimentos.

\begin{tabular}{|c|c|c|c|c|c|c|c|c|c|c|}
\hline $\begin{array}{l}\text { Profundi- } \\
\text { dade } \\
(\mathrm{cm})\end{array}$ & $\begin{array}{c}\mathrm{pH} \\
\text { em } \mathrm{CaCl}_{2}\end{array}$ & $\begin{array}{l}\text { Matéria } \\
\text { orgânica } \\
\left(\mathrm{g} \mathrm{dm}^{-3}\right)\end{array}$ & $\begin{array}{c}\text { P-resina } \\
\left(\mathrm{mg} \mathrm{dm}^{-3}\right)\end{array}$ & $\mathrm{K}$ & $\mathrm{Ca}$ & $\begin{array}{l}\mathrm{Mg} \\
(\mathrm{mm}\end{array}$ & $\begin{array}{l}\mathrm{H}+\mathrm{Al} \\
\left.-^{-3}\right)---\end{array}$ & $\mathrm{Al}$ & CTC & $\begin{array}{c}\mathrm{V} \\
(\%)\end{array}$ \\
\hline & \multicolumn{10}{|c|}{ Plantio convencional } \\
\hline $0-20$ & 6,0 & 19 & 16 & 1,1 & 42 & 18 & 17 & 0 & 78 & 78 \\
\hline $20-40$ & 5,7 & 13 & 4 & 1,1 & 28 & 12 & 18 & 0 & 59 & 70 \\
\hline $40-60$ & 5,6 & 10 & 1 & 0,9 & 18 & 9 & 20 & 0 & 48 & 58 \\
\hline & \multicolumn{10}{|c|}{ Plantio com cobertura morta } \\
\hline $0-20$ & 5,8 & 21 & 9 & 1,4 & 38 & 16 & 19 & 0 & 74 & 75 \\
\hline $20-40$ & 5,4 & 12 & 2 & 1,5 & 22 & 9 & 20 & 0 & 53 & 62 \\
\hline $40-60$ & 5,5 & 10 & 1 & 0,9 & 20 & 8 & 20 & 0 & 49 & 59 \\
\hline
\end{tabular}

${ }^{(1)} \mathrm{CTC}$ (capacidade de troca catiônica) $=\mathrm{K}+\mathrm{Ca}+\mathrm{Mg}+\mathrm{H}+\mathrm{Al} ; \mathrm{V}$ (saturação de bases $)=(\mathrm{S} / \mathrm{CTC}) \mathrm{x} 100 ; \mathrm{S}($ soma de bases $)=\mathrm{K}+\mathrm{Ca}+\mathrm{Mg}$.

nação do teor de água e posterior cálculo da matéria seca.

Os dados obtidos foram submetidos à análise de regressão e de variância da regressão, utilizando-se, respectivamente, os procedimentos REG e GLM do pacote estatístico SAS (SAS Institute, 1993). As produções de cada experimento foram analisadas a partir do modelo polinomial quadrático, que permite o ajuste de funções da superfície de resposta do tipo $\mathrm{Y}=\mathrm{b}_{0}+\mathrm{b}_{1} \mathrm{~N}+\mathrm{b}_{2} \mathrm{~N}^{2}+\mathrm{b}_{3} \mathrm{P}+\mathrm{b}_{4} \mathrm{P}^{2}+\mathrm{b}_{5} \mathrm{~K}$ $+b_{6} K^{2}+b_{7} N P+b_{8} N K+b_{9} P K$, em que Y: produção de matéria seca de forragem $\left(\mathrm{t} \mathrm{ha}^{-1}\right)$; b: coeficiente de regressão; $\mathrm{N}, \mathrm{Pe} \mathrm{K}$ : doses de $\mathrm{N}, \mathrm{P}_{2} \mathrm{O}_{5}$ e $\mathrm{K}_{2} \mathrm{O}$, em kg ha ${ }^{-1}$, respectivamente.

As doses e as combinações de nutrientes para máxima produção foram obtidas por meio do cálculo diferencial $\partial \mathrm{Y} / \partial \mathrm{N}=0, \partial \mathrm{Y} / \partial \mathrm{P}=0$ e $\partial \mathrm{Y} / \partial \mathrm{K}=0$, e para o lucro máximo, calculadas por $\partial \mathrm{Y} / \partial \mathrm{N}=\mathrm{V} / \mathrm{C}_{\mathrm{N}}, \partial \mathrm{Y} / \partial \mathrm{P}=\mathrm{V} / \mathrm{C}_{\mathrm{P}} \mathrm{e}$ $\partial \mathrm{Y} / \partial \mathrm{K}=\mathrm{V} / \mathrm{C}_{\mathrm{K}}$, em que $\mathrm{V}$ é o preço de $1 \mathrm{~kg}$ de matéria seca de forragem; $\mathrm{C}_{\mathrm{N}}, \mathrm{C}_{\mathrm{P}}$ e $\mathrm{C}_{\mathrm{K}}$ representam preço de $1 \mathrm{~kg}$ de $\mathrm{N}$, $\mathrm{P}_{2} \mathrm{O}_{5}$ e $\mathrm{K}_{2} \mathrm{O}$, respectivamente. Nos cálculos do retorno econômico foram considerados os seguintes preços: feno de aveia $=\mathrm{R} \$ 0,21 / \mathrm{kg} ; \mathrm{N}=\mathrm{R} \$ 1,09 / \mathrm{kg} ; \mathrm{P}_{2} \mathrm{O}_{5}=\mathrm{R} \$ 1,19 / \mathrm{kg} \mathrm{e}$ $\mathrm{K}_{2} \mathrm{O}=\mathrm{R} \$ 0,75 / \mathrm{kg}$.

\section{Resultados e Discussão}

A análise de variância revelou resposta apenas para o N nos dois sistemas de plantio. Foi observada interação NxP positiva significativa, mas apenas para o plantio com cobertura morta.

No plantio convencional, as produções de forragem de aveia nas doses máximas utilizadas de $\mathrm{N}$ sugerem proximidade do ponto de máxima, ao passo que no plantio com cobertura morta os dados indicam que a produção poderia aumentar ainda mais com doses maiores desse nutriente (Tabelas 2 e 3). Frizzone et al. (1995), trabalhando com aveia, obtive-
Tabela 2. Produção estimada de matéria seca de forragem de aveia em resposta à adubação com $\mathrm{N}, \mathrm{P}_{2} \mathrm{O}_{5}$ e $\mathrm{K}_{2} \mathrm{O}$ em dois sistemas de plantio.

\begin{tabular}{|c|c|c|c|c|}
\hline $\mathrm{N}$ & $\mathrm{P}_{2} \mathrm{O}_{5}$ & $\mathrm{~K}_{2} \mathrm{O}$ & $\begin{array}{c}\text { Plantio } \\
\text { convencional }\end{array}$ & $\begin{array}{c}\text { Plantio com } \\
\text { cobertura morta }\end{array}$ \\
\hline \multirow{2}{*}{$--\cdot$} & & -.---.. & $\left.\mathrm{g} \mathrm{ha}^{-1}\right)$ & --------------- \\
\hline & 0 & 0 & 2.271 & 2.211 \\
\hline 0 & 0 & 210 & 2.049 & 2.158 \\
\hline 0 & 60 & 70 & 2.634 & 2.469 \\
\hline 0 & 60 & 140 & 2.733 & 2.412 \\
\hline 0 & 120 & 70 & 3.175 & 2.401 \\
\hline 0 & 120 & 140 & 3.075 & 2.772 \\
\hline 0 & 180 & 0 & 2.809 & 2.875 \\
\hline 0 & 180 & 210 & 2.711 & 2.155 \\
\hline 70 & 0 & 70 & 5.340 & 4.549 \\
\hline 70 & 0 & 140 & 5.285 & 4.818 \\
\hline 70 & 60 & 0 & 4.994 & 4.572 \\
\hline 70 & 60 & 210 & 5.091 & 4.531 \\
\hline 70 & 120 & 0 & 5.197 & 5.402 \\
\hline 70 & 120 & 210 & 5.136 & 4.331 \\
\hline 70 & 180 & 70 & 4.982 & 5.134 \\
\hline 70 & 180 & 140 & 5.601 & 4.611 \\
\hline 140 & 0 & 70 & 6.166 & 5.667 \\
\hline 140 & 0 & 140 & 6.120 & 5.433 \\
\hline 140 & 60 & 0 & 6.117 & 6.025 \\
\hline 140 & 60 & 210 & 7.187 & 5.929 \\
\hline 140 & 120 & 0 & 6.486 & 6.814 \\
\hline 140 & 120 & 210 & 6.466 & 6.663 \\
\hline 140 & 180 & 70 & 5.862 & 4.819 \\
\hline 140 & 180 & 140 & 6.272 & 7.050 \\
\hline 210 & 0 & 0 & 5.474 & 6.037 \\
\hline 210 & 0 & 210 & 6.056 & 6.880 \\
\hline 210 & 60 & 70 & 7.184 & 7.559 \\
\hline 210 & 60 & 140 & 6.880 & 7.460 \\
\hline 210 & 120 & 70 & 7.424 & 7.638 \\
\hline 210 & 120 & 140 & 6.904 & 8.234 \\
\hline 210 & 180 & 0 & 6.814 & 7.684 \\
\hline 210 & 180 & 210 & 7.437 & 7.792 \\
\hline Média & & & 5.248 & 5.159 \\
\hline Teste F & & & $\mathrm{N}_{\mathrm{L}}^{* *} \mathrm{~N}_{\mathrm{Q}}^{* *}$ & $\begin{array}{c}\mathrm{N}_{\mathrm{L}}^{* *} \mathrm{~N}_{\mathrm{Q}}^{* *} \\
\mathrm{NxP}^{*}\end{array}$ \\
\hline CV (\%) & & & 7,4 & 7,8 \\
\hline
\end{tabular}


ram resposta quadrática à aplicação de $\mathrm{N}$ com um valor máximo estimado de produção de $6,9 \mathrm{t} \mathrm{ha}^{-1} \mathrm{com}$ a dose de $152 \mathrm{~kg} \mathrm{ha}^{-1}$ de N. Já Lupatini et al. (1998), avaliando mistura de aveia e azevém sob pastejo submetida a níveis de $\mathrm{N}$, verificaram aumentos lineares de produção de matéria seca, com o acréscimo das doses de $\mathrm{N}$, dentro dos limites estudados $(0 \mathrm{a}$ $300 \mathrm{~kg} \mathrm{ha}^{-1}$ de N).

Houve um decréscimo na produção de forragem de aveia no quarto corte nos dois sistemas de plantio (Tabela 3). Provavelmente tal fato tenha ocorrido por causa da idade fisiológica da planta, que no último corte já estava finalizando o seu ciclo. $\mathrm{O} \mathrm{N}$ foi o fator mais limitante dessa produção.

O solo onde o plantio foi convencional apresentava teores médios de P-resina, provavelmente por esse motivo não houve resposta à aplicação desse nutriente. A faixa de valores baixos de P-resina para culturas anuais é de 0 a $15 \mathrm{mg} \mathrm{dm}^{-3}$, e de valores médios de 16 a $40 \mathrm{mg} \mathrm{dm}^{-3}$, conforme Raij et al. (1996).

No plantio no solo com cobertura morta, as respostas às doses de $\mathrm{P}_{2} \mathrm{O}_{5}$ em produção de matéria seca aumentaram com o acréscimo das doses de $\mathrm{N}$ (Tabela 4).

Embora não tenha havido resposta ao $\mathrm{P}$, mesmo com seu teor disponível estando na faixa dos teores baixos (Tabela 1), a interação entre N e P provavelmente reflete os rendimentos mais altos. Por sua vez, Carriel et al. (1984) constataram que o $\mathrm{P}$ e o $\mathrm{N}$ foram os nutrientes que mais limitaram o estabelecimento, a produção de matéria seca e o perfilhamento de aveia.
As equações relacionando produção de forragem e doses de $\mathrm{N}, \mathrm{P}_{2} \mathrm{O}_{5}$ e $\mathrm{K}_{2} \mathrm{O}$ aplicadas nos dois sistemas de plantio, com indicação de significância dos coeficientes, foram: $\mathrm{Y}=2.390+38,79 \mathrm{~N}-0,1063 \mathrm{~N}^{2(* *)}$ $+9,582 \mathrm{P}-0,047 \mathrm{P}^{2}+3,96 \mathrm{~K}-0,0213 \mathrm{~K}^{2}+0,012984 \mathrm{NP}+$ $0,014379 \mathrm{NK}+0,000869 \mathrm{PK}$, no plantio convencional, e $\mathrm{Y} 1=2.359+28,93 \mathrm{~N}-0,0486 \mathrm{~N}^{2(* *)}+10,37 \mathrm{P}-$ $0,0463 \mathrm{P}^{2}-0,0815 \mathrm{~K}-0,0062 \mathrm{~K}^{2}+0,024792 \mathrm{NP}^{(*)}+$ $0,021333 \mathrm{NK}-0,011586 \mathrm{PK}$, no plantio com cobertura morta.

Apesar da significância estatística apenas em relação ao N, optou-se por considerar a equação com os três elementos para a análise econômica, pois o coeficiente de determinação diminuiu muito com o modelo ajustado apenas para o nitrogênio.

No caso do plantio convencional, pela equação do ajuste (Y), verificou-se que, para cada $\mathrm{kg}$ de $\mathrm{K}_{2} \mathrm{O}$ aplicado, houve aumento de rendimento de $3,96 \mathrm{~kg}$ de matéria seca de aveia. Como a relação de preços K/aveia foi muito próxima do aumento de rendimento, pois são necessários 3,57 kg de aveia para pagar $1 \mathrm{~kg}$ de $\mathrm{K}_{2} \mathrm{O}$, somente compensa adubar com K para manter o nível desse nutriente no solo. $\mathrm{Na}$ área do plantio convencional, a análise do solo indicou teores baixos de K, e como a resposta à aplicação desse nutriente foi pequena, indica que essa cultura é muito eficiente na extração de $\mathrm{K}$ do solo. Os coeficientes lineares de $\mathrm{N}$ e $\mathrm{P}(\mathrm{N}=38,8$ e $\mathrm{P}=9,6)$ também foram maiores que a relação de preços $(\mathrm{N}=5,2$ e $\mathrm{P}=5,7)$.

Tabela 3. Produção média estimada de matéria seca de forragem de aveia por corte $(C)$ e total de quatro cortes, em dois sistemas de plantio, em resposta à adubação com $\mathrm{N}, \mathrm{P}_{2} \mathrm{O}_{5}$ e $\mathrm{K}_{2} \mathrm{O}^{(1)}$.

\begin{tabular}{|c|c|c|c|c|c|c|c|c|c|c|}
\hline \multirow[t]{2}{*}{ Doses } & \multicolumn{5}{|c|}{ Convencional } & \multicolumn{5}{|c|}{ Com cobertura } \\
\hline & $1^{\circ} \mathrm{C}$ & $2^{\circ} \mathrm{C}$ & $3^{\circ} \mathrm{C}$ & $4^{\circ}-\mathrm{C}$ & Total & $1^{\circ} \mathrm{C}$ & $2^{\circ} \mathrm{C}$ & $3^{\circ} \mathrm{C}$ & $4^{\circ} \mathrm{C}$ & Total \\
\hline & \multicolumn{10}{|c|}{$\mathrm{N}$} \\
\hline 0 & 687 & 775 & 815 & 405 & 2.682 & 462 & 821 & 793 & 355 & 2.431 \\
\hline 70 & 1.160 & 1.870 & 1.384 & 789 & 5.203 & 793 & 1.723 & 1.459 & 770 & 4.744 \\
\hline 140 & 1.389 & 2.699 & 1.380 & 867 & 6.334 & 1.003 & 2.557 & 1.728 & 762 & 6.050 \\
\hline \multirow[t]{2}{*}{210} & 1.551 & 2.905 & 1.341 & 974 & 6.772 & 1.269 & 3.287 & 2.012 & 843 & 7.411 \\
\hline & \multicolumn{10}{|c|}{$\mathrm{P}_{2} \mathrm{O}_{5}$} \\
\hline 0 & 977 & 1.996 & 1.223 & 649 & 4.851 & 788 & 1.880 & 1.496 & 556 & 4.719 \\
\hline 60 & 1.191 & 2.129 & 1.237 & 796 & 5.352 & 886 & 2.072 & 1.468 & 694 & 5.200 \\
\hline 120 & 1.291 & 2.023 & 1.282 & 741 & 5.483 & 977 & 2.185 & 1.527 & 843 & 5.532 \\
\hline \multirow[t]{2}{*}{180} & 1.328 & 2.101 & 1.178 & 850 & 5.311 & 876 & 2.251 & 1.502 & 637 & 5.265 \\
\hline & \multicolumn{10}{|c|}{$\mathrm{K}_{2} \mathrm{O}$} \\
\hline 0 & 1.125 & 1.920 & 1.084 & 891 & 5.020 & 925 & 2.163 & 1.505 & 610 & 5.203 \\
\hline 70 & 1.275 & 1.946 & 1.224 & 902 & 5.346 & 891 & 2.050 & 1.410 & 679 & 5.030 \\
\hline 140 & 1.181 & 2.210 & 1.293 & 676 & 5.359 & 917 & 2.108 & 1.553 & 771 & 5.349 \\
\hline 210 & 1.206 & 2.173 & 1.319 & 568 & 5.267 & 794 & 2.067 & 1.524 & 670 & 5.055 \\
\hline
\end{tabular}

(1) Data dos cortes: 1으 $\mathrm{C}=7 / 7 / 2000 ; 2$ 으 $\mathrm{C}=11 / 8 / 2000 ; 3$ ㅇ $\mathrm{C}=15 / 9 / 2000$; 4ㅇ $\mathrm{C}=20 / 10 / 2000$. 
No plantio com cobertura morta, os coeficientes lineares de $\mathrm{N}$ e $\mathrm{P}(\mathrm{N}=28,93$ e $\mathrm{P}=10,4)$ foram maiores que a relação de preços, mas em relação ao $\mathrm{K}$, o coeficiente linear foi negativo e, portanto, não houve resposta à adição deste nutriente, apesar do seu teor no solo se encontrar na faixa de valores baixos. Provavelmente, na área com cobertura morta parte do $\mathrm{K}$ foi fornecido pela palhada, além de essa cultura ser eficiente na extração desse nutriente do solo.

Constatou-se que, na determinação da produção máxima e da receita líquida máxima, ocorreu extrapolação das doses de nutrientes, provavelmente pelas limitações no ajuste das equações. Desta forma, as estimativas das doses de nutrientes para maior produtividade e retorno econômico (Tabela 5) ficaram restritas aos limites dos tratamentos utilizados, conforme recomendação de Pimentel-Gomes \& Conagin (1991).

As doses de fertilizantes para máximo retorno econômico da aveia para produção de forragem, em quatro cortes consecutivos e com irrigação, foram de 165,50 e $53 \mathrm{~kg} \mathrm{ha}^{-1}$ de $\mathrm{N}, \mathrm{P}_{2} \mathrm{O}_{5}$ e $\mathrm{K}_{2} \mathrm{O}$, respectivamente, no cultivo convencional, e de 210 e $90 \mathrm{~kg} \mathrm{ha}^{-1}$ de $\mathrm{N}$ e $\mathrm{P}_{2} \mathrm{O}_{5}$, respectivamente, no sistema com cobertura morta (Tabela 5). Esses valores são maiores do que os recomendados para a produção de grãos de aveia em condições de sequeiro no Estado de São

Tabela 4. Produção estimada de matéria seca de forragem de aveia com as doses de $\mathrm{N}$ e de $\mathrm{P}_{2} \mathrm{O}_{5}$, no plantio com cobertura morta.

\begin{tabular}{|c|c|c|c|c|}
\hline \multirow[t]{3}{*}{$\mathrm{P}_{2} \mathrm{O}_{5}$} & \multicolumn{4}{|c|}{ Nitrogênio } \\
\hline & 0 & 70 & 140 & 210 \\
\hline & ---------- & ------ & $\left.a^{-1}\right)---$ & 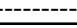 \\
\hline 0 & 2.185 & 4.684 & 5.550 & 6.459 \\
\hline 60 & 2.440 & 4.552 & 5.977 & 7.509 \\
\hline 120 & 2.587 & 4.867 & 6.739 & 7.936 \\
\hline 180 & 2.515 & 4.873 & 5.934 & 7.738 \\
\hline
\end{tabular}

Tabela 5. Doses de $\mathrm{N}, \mathrm{P}_{2} \mathrm{O}_{5}$ e $\mathrm{K}_{2} \mathrm{O}$ para máxima produção agronômica e máxima receita líquida de forragem de aveia.

\begin{tabular}{|c|c|c|c|c|c|c|}
\hline \multirow[t]{3}{*}{$\begin{array}{l}\text { Sistemas } \\
\text { de plantio }^{(1)}\end{array}$} & \multicolumn{3}{|c|}{$\begin{array}{l}\text { Máxima produção } \\
\text { agronômica }\end{array}$} & \multicolumn{3}{|c|}{$\begin{array}{l}\text { Máxima receita } \\
\text { líquida }^{(2)}\end{array}$} \\
\hline & $\mathrm{N}$ & $\mathrm{P}_{2} \mathrm{O}_{5}$ & $\mathrm{~K}_{2} \mathrm{O}$ & $\mathrm{N}$ & $\mathrm{P}_{2} \mathrm{O}_{5}$ & $\mathrm{~K}_{2} \mathrm{O}$ \\
\hline & & & & & & \\
\hline Conv & 201 & 13 & 161 & 165 & 50 & 53 \\
\hline Com cobertura & 210 & 142 & 210 & 210 & 90 & 0 \\
\hline
\end{tabular}

(1)Plantio convencional: produção máxima de $7.235 \mathrm{~kg} \mathrm{ha}^{-1}$ e produção na dose de $\mathrm{N}, \mathrm{P}_{2} \mathrm{O}_{5}$ e $\mathrm{K}_{2} \mathrm{O}$ mais econômica de $6.641 \mathrm{~kg} \mathrm{ha}^{-1}$; plantio com cobertura: produção máxima de $7.878 \mathrm{~kg} \mathrm{ha}^{-1}$ e produção na dose de $\mathrm{N}$, $\mathrm{P}_{2} \mathrm{O}_{5}$ e $\mathrm{K}_{2} \mathrm{O}$ mais econômica de $7.322 \mathrm{~kg} \mathrm{ha}^{-1}$. ${ }^{(2)}$ Calculada com os seguintes preços $\left(\mathrm{R}_{\mathrm{kg}}{ }^{-1}\right): \mathrm{N}=1,09 ; \mathrm{P}_{2} \mathrm{O}_{5}=1,19 ; \mathrm{K}_{2} \mathrm{O}=0,75$ e feno de aveia $=0,21$.
Paulo, cujas doses máximas são de 70, 90 e $60 \mathrm{~kg} \mathrm{ha}^{-1}$ de N, $\mathrm{P}_{2} \mathrm{O}_{5}$ e $\mathrm{K}_{2} \mathrm{O}$, respectivamente (Camargo et al., 1996). No entanto, deve-se levar em conta que a exportação de nutrientes pelos grãos, para rendimento de $3 \mathrm{t} \mathrm{ha}^{-1}$ está em torno de 60, 21 e $25 \mathrm{~kg} \mathrm{ha}^{-1} \mathrm{de} \mathrm{N}$, $\mathrm{P}_{2} \mathrm{O}_{5}$ e K $\mathrm{K}_{2} \mathrm{O}$, respectivamente (Cantarella et al., 1996), ao passo que, para produzir cerca de $7 \mathrm{t} \mathrm{ha}^{-1}$ de matéria seca de aveia, são exportados aproximadamente 175,35 e $253 \mathrm{~kg} \mathrm{ha}^{-1}$ de $\mathrm{N}, \mathrm{P}_{2} \mathrm{O}_{5}$ e $\mathrm{K}_{2} \mathrm{O}$, respectivamente (Primavesi \& Primavesi, 2001).

Nos dois sistemas de plantio, a resposta ao $\mathrm{N}$ apresentou o maior retorno em produção de forragem por unidade de nutriente aplicado. No plantio com cobertura morta, a eficiência do $\mathrm{N}$ foi menor, provavelmente decorrente de sua imobilização parcial pelos microorganismos do solo, durante a oxidação da palhada. Segundo Salet et al. (1997), a principal causa da menor disponibilidade de $\mathrm{N}$ no sistema de plantio direto é a imobilização microbiana do $\mathrm{N}$ quando o fertilizante nitrogenado é aplicado em cobertura.

\section{Conclusões}

1. O potencial de produção de forragem de aveia linhagem UPF 87111 é maior em sistemas de plantio com cobertura morta.

2. No sistema de plantio convencional, a máxima receita líquida para produção de $6,6 \mathrm{t} \mathrm{ha}^{-1}$ de forragem de aveia é obtida com a aplicação de 165, 50 e $53 \mathrm{~kg} \mathrm{ha}^{-1}$ de $\mathrm{N}, \mathrm{P}_{2} \mathrm{O}_{5}$ e $\mathrm{K}_{2} \mathrm{O}$, respectivamente.

3. No sistema de plantio com cobertura morta, a máxima receita líquida para produção de $7,3 \mathrm{t} \mathrm{ha}^{-1} \mathrm{de}$ forragem de aveia é obtida com a aplicação de 210 e $90 \mathrm{~kg} \mathrm{ha}^{-1} \mathrm{de} \mathrm{N}, \mathrm{P}_{2} \mathrm{O}_{5}$, respectivamente.

\section{Referências}

ANDRADE, D. F.; NOLETO, A. Q. Exemplos de fatoriais fracionados $(1 / 2) 4^{3}$ e $(1 / 4)^{4}$ para o ajuste de modelos polinomiais quadráticos. Pesquisa Agropecuária Brasileira, Brasília, v. 21, n. 6, p. 677-680, jun. 1986.

BACCHI, O. O. S.; GODOY, R.; FANTE JÚNIOR, L.; REICHARDT, K. Balanço hídrico em cultura de aveia forrageira de inverno na região de São Carlos, SP. Scientia Agricola, Piracicaba, v. 53, n. 1, p. 172-178, jan./ abr. 1996. 
CAMARGO, C. E. O.; FREITAS, J. G.; CANTARELLA, H. Aveia e centeio. In: RAIJ, B. van; CANTARELLA, H.; QUAGGIO, J. A.; FURLANI, A. M. C. (Ed.). Recomendações de adubação e calagem para o Estado de São Paulo. Campinas: Instituto Agronômico, 1996. p. 52-53. (Boletim Técnico, 100).

CANTARELLA, H.; RAIJ, B. van; CAMARGO, C. E. O. Cereais. In: RAIJ, B. van; CANTARELLA, H.; QUAGGIO, J. A.; FURLANI, A. M. C. (Ed.). Recomendações de adubação e calagem para o Estado de São Paulo. Campinas: Instituto Agronômico, 1996. p. 45-71. (Boletim Técnico, 100).

CARRIEL, R. E.; COLOZZA, M. T.; PAULINO, V. T. Limitações nutricionais de dois solos de várzeas do Estado de São Paulo para o cultivo de aveia e de azevém. Zootecnia, Nova Odessa, v. 22, p. 153-177, 1984.

FRIZZONE, J. A.; TEODORO, R. E. F.; PEREIRA, A. S.; BOTREL, T. A. Lâminas de água e doses de nitrogênio na produção de aveia (Avena sativa L.) para forragem. Scientia Agricola, Piracicaba, v. 52, p. 578-586, 1995.

GODOY, R.; RODRIGUES, L. R. A.; REIS, R. A.; HERLING, V. R.; SILVA, J. R.; SMITH, M. F.; PRIMAVESI, A. C. A.; BATISTA, L. A. R. Recomendação de cultivares de aveia para a produção de forragem no Estado de São Paulo. São Carlos: Embrapa-CPPSE, 1999. 7 p. (Comunicado Técnico, 19).

LUPATINI, G. C.; RESTle, J.; CERETTA, M.; MOOJEN, E. L.; BARTZ, H. R. Avaliação da mistura de aveia preta e azevém sob pastejo submetida a níveis de nitrogênio. Pesquisa Agropecuária Brasileira, Brasília, v. 33, n. 11, p. 1939-1943, nov. 1998.

PIMENTEL-GOMES, F.; CONAGIN, A. Experimentos de adubação: planejamento e análise estatística. In: OLIVEIRA, A. J. de; GARRIDO, W. E.; ARAÚJO, J. D. de; LOURENÇO, S. (Coord.). Métodos de pesquisa em fertilidade do solo. Brasília: Embrapa-SEA, 1991.392 p. (Documentos, 3).

PRIMAVESI, A. C.; GODOY, R.; PRIMAVESI, O. Aveia forrageira: épocas de corte. São Carlos: Embrapa-CPPSE, 2000a. 10 p. (Comunicado Técnico, 30).
PRIMAVESI, A. C.; GODOY, R.; PRIMAVESI, O.; PEDROSO, A. F. Manejo de aveia forrageira. In: SEMANA DO ESTUDANTE, 13., 1999, São Carlos. Anais... São Carlos: Embrapa-CPPSE, 1999. p. 130-140.

PRIMAVESI, A. C.; PRIMAVESI, O. Efeito de intervalos de corte e de épocas de plantio sobre os teores de minerais em cultivares de aveia. Revista de Agricultura, Piracicaba, v. 76, p. 3-18, 2001.

PRIMAVESI, A. C.; PRIMAVESI, O.; CHINELLATO, A.; GODOY, R. Indicadores de determinação de cortes de cultivares de aveia forrageira. Scientia Agricola, Piracicaba, v. 58, p. $79-89,2001$.

PRIMAVESI, A. C. P. A.; PRIMAVESI, O.; GODOY, R. Manejo de cortes de cultivares de aveia forrageira. In: REUNIẪO ANUALDA SOCIEDADE BRASILEIRA DE ZOOTECNIA, 37., 2000, Viçosa, MG. Anais... Viçosa, MG: UFV, 2000b. 3 p. Seção Oral. CD-ROM.

RAIJ, B. van; QUAGGIO, J. A.; CANTARELLA, H.; ABREU, C. A. Interpretação de resultados de análise de solo. In: RAIJ, B. van; CANTARELLA, H.; QUAGGIO, J. A.; FURLANI, A. M. C. (Ed.). Recomendações de adubação e calagem para o Estado de São Paulo. Campinas: Instituto Agronômico, 1996. p. 8-13. (Boletim Técnico, 100).

RAIJ, B. van; QUAGGIO, J. A.; CANTARELLA, H.; FERREIRA, M. E.; LOPES, A. S.; BATAGLIA, O. Análise química do solo para fins de fertilidade. Campinas: Fundação Cargill, 1987. 170 p.

RESENDE, M. Clima do solo e suas relações com o ambiente agrícola. Informe Agropecuário, Belo Horizonte, v. 12, p. 43-59, 1986.

SALET, R. L.; VARGAS, L. K.; ANGHINONI, I.; KOCHHANN, R. A.; DENARDIN, J. E.; CONTE, E. Por que a disponibilidade de nitrogênio é menor no sistema plantio direto? In: SEMINÁRIO INTERNACIONAL DO SISTEMA PLANTIO DIRETO, 2., 1997, Passo Fundo. Anais... Passo Fundo: Embrapa-CNPT, 1997. p. 217-219.

SAS INSTITUTE (Cary, Estados Unidos). SAS/STAT user's guide: statistics, version 6.4. ed. Cary, 1993. v. 2.

SEDIYAMA, G. C.; PRATES, J. E. O microclima: possibilidades de modificação. Informe Agropecuário, Belo Horizonte, v. 12, p. 38-42, 1986. 